\title{
The limbus: Structure and Function
}

\begin{abstract}
Limbal function is a key determinant of corneal epithelial integrity. Lineage tracing studies in mice have highlighted that the centripetal movement of epithelial progenitors from the limbus drives both the steady-state maintenance of the corneal epithelium and its regeneration following injury. It is well established that this is facilitated by a population of limbal epithelial stem cells within the limbus. It is becoming increasingly apparent that the behaviour of these stem cells and their ability to respond to the needs of the tissue are closely linked to their immediate microenvironment - the stem cell niche. Increasing understanding of the structural features of this niche and the signalling networks that they coordinate is required to enhance the therapeutic application of these cells in the treatment of limbal stem cell deficiency. Importantly, an improved characterisation of the hierarchy of limbal epithelial progenitors using both new and old putative markers will enable a greater appreciation for the effects of many of these limbal niche factors on stem cell fate.
\end{abstract}

\author{
Authors \\ Ashkon G. Seyed-Safi ${ }^{1,2,3}$, Julie T. Daniels ${ }^{1}$. \\ ${ }^{1}$ UCL Institute of Ophthalmology, ${ }^{2}$ UCL Medical School. \\ ${ }^{3}$ Corresponding author: ashkon.seyed-safi.16@ucl.ac.uk \\ Address: UCL Institute of Ophthalmology, 11-43 Bath street, EC1V 9EL, London, UK
}

Declaration of interests: none. 


\section{Introduction}

The cornea overlies the anterior chamber of the eye and is a transparent tissue that is vital for the transmission and focusing of light on to the retina, as well as providing protection to the internal structures of the eye. The proper functioning of all three cellular layers of the cornea, the epithelium, stroma, and epithelium, is required to preserve this function. The limbus can be easily identified as an annular transition zone on the surface of the eye, sitting between the transparent cornea and adjacent conjunctiva (Van Buskirk, 1989). Limbal integrity is a key determinant of maintaining a clear, avascular cornea and this relationship is observed clinically in limbal stem cell deficiency (LSCD) (Huang and Tseng, 1991; Le et al., 2018). It is well documented that following acute or ongoing damage to limbal tissue there is a loss of corneal epithelial integrity, often characterised by the invasion of the corneal surface by conjunctival epithelium and blood vessels.

It is widely accepted that a population of unipotent epithelial stem cells exists within the limbus (LESCS). These cells are thought to be uniquely responsible for maintaining the corneal epithelium under homeostatic conditions, and also in response to tissue injury (Cotsarelis et al., 1989; Lehrer et al., 1998). However, the exclusive role of the limbus in this function has been challenged in recent years. This has in turn provoked renewed efforts to provide more conclusive evidence of the exact function of limbal derived cells in maintaining and regenerating the corneal surface.

In addition to clarifying the function of LESCs, it is important to look at the ultrastructure of the limbus to delineate their position as well as their immediate microenvironment (Yazdanpanah et al., 2019). The stem cell niche is an established concept, and a more comprehensive understanding of the relationship between the stem cells and their niche has important implications for our ability to harness these cells therapeutically.

LESCs have long represented an attractive population to study, in particular due to the fact that limbus in which these cells are enriched can be easily identified on the ocular surface. However, despite this, these cells present an enormous challenge which is the fact that over the last few decades, no single marker or assay has been agreed upon as a defining feature of bona fide LESCs (Guo et al., 2018).

Thus, the precise function of the limbus with respect to maintaining the corneal epithelium, and the structure of the niche microenvironment in which LESCs may be preserved, still represent important and dynamic topics of investigation. In this review, the focus will be on recent studies that have provided further insight into these questions on the limbus. We must also consider the significant barrier that the lack of a universal stem cell marker has been. Therefore, recent efforts to improve our understanding of limbal stem cell hierarchy and how this relates to the expression of canonical and novel markers will also be explored. 


\section{The ABCs of the $X Y Z$ hypothesis - Limbal function}

\subsection{Role of the limbus in epithelial maintenance:}

The corneal epithelium has a remarkable turnover rate, and it is estimated that under homeostatic conditions it is regenerated every 5-14 days with variability across species (Cenedella and Fleschner, 1990; Haddad, 2000; Hanna and O'brien, 1960). Thoft and Friend described the process of replacing damaged or desquamated cells in their XYZ hypothesis (Thoft and Friend, 1983). They proposed that $X+Y=Z$, wherein $X$ represents the movement of cells superficially from the basal epithelium, $Y$ the centripetal migration of basal cells from the limbus. Together these two processes maintain tissue mass by replacing the cells lost from the surface by desquamation or injury, represented as $Z$.

Underpinning this dynamic relationship is the concept that LESCs, in the basal layer of the limbus, are responsible for dividing to generate the progenitors to repopulate the corneal epithelium. As the progenitors, termed transient amplifying cells, migrate centripetally and pass anteriorly through to the wing and squamous layers of the epithelium, they become terminally differentiated cells.

One series of experiments offered an alternative hypothesis (Majo et al., 2008). It was proposed that in addition to those in the limbus, stem cells exist throughout that mammalian ocular surface, including the central cornea. The major evidence for this is the observation that when limbal sections from $\beta$-gal-ROSA26 mice were surgically transferred to the limbus of SCID mice, labelled cells from the transplanted tissue did not appear to contribute to the maintenance of the epithelial surface. In contrast, labelled corneal tissue transplanted to both the limbal and central corneal regions of recipient mice did appear to contribute to the epithelial surface. It is unclear what influence the surgical manipulation had, and also since only partial sections limbal tissue were transplanted, it is entirely possible that other regions of the limbus may have compensated. Opposition to this alternative hypothesis, citing incompatibility of the conclusions with the wider literature has been presented elsewhere (Sun et al., 2010).

Multiple lineage tracing studies have since been published, in order to track the contribution of LESCs to epithelial maintenance. Two independent groups used confetti mouse models, in which $\mathrm{CK} 14^{+}$epithelial cells, shown to be enriched in the limbus, were tracked in mice over a period of months (Amitai-Lange et al., 2015; Di Girolamo et al., 2015). In both instances' streaks were observed extending from the limbus to the centre, demonstrating the contribution of the limbal progenitors to maintenance of the corneal epithelium. An alternative unbiased non-progenitor-specific labelling approach has also been reported, in which mice were injected with tamoxifen to induce LacZ reporter gene expression and stained for $\beta$-galactosidase (Dorà et al., 2015). Cells were labelled across the ocular surface, but short-lived clones were shed during the chase period. In contrast, longerlived clones, believe to represent the progenitor population remained throughout the chase period. Once again, streaks originating from the limbus were observed extending centrally. Together these studies provide strong evidence that the long-term steady state maintenance of the corneal epithelium is primarily a function of the limbus. However, it remains to be seen whether similar processes can be demonstrated experimentally in other species with a more similar physiology to humans. 
2.2 Role of the limbus in epithelial wound healing:

There has long been evidence to suggest that the limbus is stimulated to mount a response following wounding (Cotsarelis et al., 1989), and Amitai-Lange and colleagues observed an 8 -fold increase in the rate of streak development in their confetti mouse model following moderate and severe corneal wounding (Amitai-Lange et al., 2015). Furthermore, data from our own group using a porcine ex vivo organ cultured wounding model demonstrated that following the generation of an $8 \mathrm{~mm}$ central debridement, there was an increase in proliferating peripheral and limbal basal epithelial cells from 12 hours post-wounding (Figure 1) (Paper accepted for publication.

A

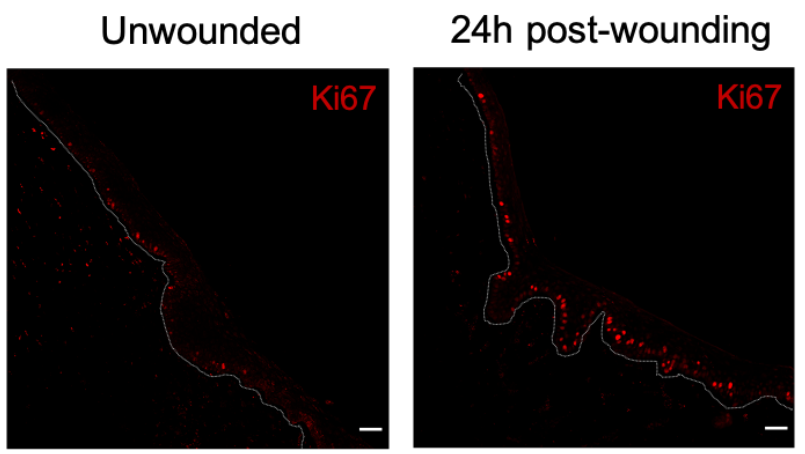

B

Ki67

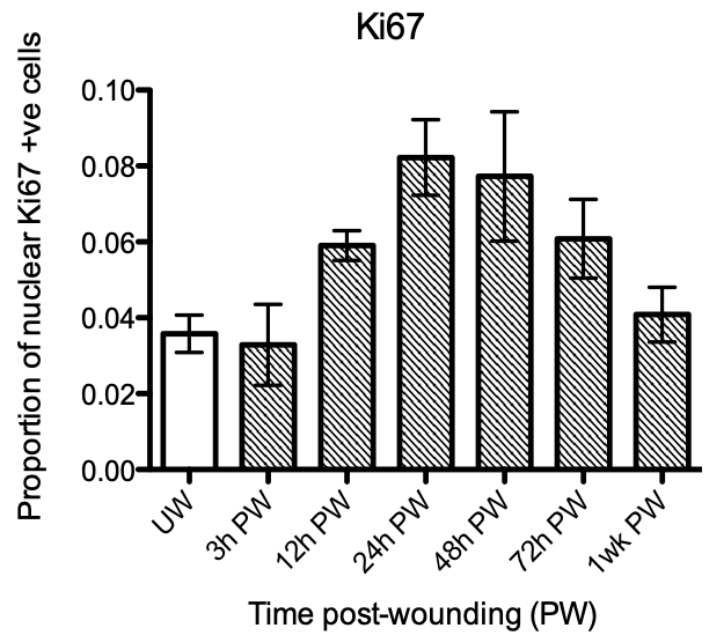

Figure 1

Following the creation of an $8 \mathrm{~mm}$ central epithelial wound, eyes were frozen and sectioned at different time points (A) Side by side of the Ki67 staining pattern in the limbal section of unwounded tissue, and limbal tissue frozen at $24 \mathrm{~h}$ post-wounding. Dotted line indicates junction between the epithelium and stroma (B) Sections taken from tissues ( $n=3$ at each time point) at a range of time points up to one week, were stained for Ki67, and the proportion of all epithelial cells that demonstrated positive nuclear staining for Ki67 were counted. Scalebar $=50 \mu \mathrm{m}$. (Figure reproduced with permission (Seyed-Safi and Daniels, 2020)) 
However, while the wounding response in these experiments would appear to be driven by a limbal response, one study also reported that the healing of small wounds instead involved the increase in the size of central corneal clusters of cells (Amitai-Lange et al., 2015). These results, while not conclusive, raises the question of whether the central cornea does indeed exhibit some latent proliferative potential, or at least whether some aspects of epithelial maintenance might be wholly independent of limbal involvement.

Confetti mice were subjected to annular debridement to directly compare the relative contributions of central and limbal epithelia to the repopulation of a wound separating the two regions (Park et al., 2019). Ex vivo organ culture of enucleated eyes allowed the creation of vector flow-maps which showed that while confetti-clones appeared to acquire both centrifugal and centripetal motion, the overall centripetal migration was faster than the centripetal movement. Longer term follow-up with intravital microscopy showed that while disjointed streaks were observed soon after injury, these disappeared suggesting that cells migrating centripetally were replacing cells from the central island. Post-wounding, the central epithelium was shown to be thinner with fewer layers of cells. However, unlike peripheral and limbal cells, BrdU uptake in cells from the central island was rare, and not found to increase significantly following wounding. Therefore, the authors suggest that centripetally migrating cells offer greater contribution to wound closure, while the slower contribution of cells from the central island is likely due to alterations in size and shape, rather than proliferation.

With respect to the mechanism of wound closure, evidence has pointed towards 'cellrolling' and 'cell-sliding' mechanisms for wound-edge movement to cover the exposed wound surface (Crosson et al., 1986; Danjo and Gipson, 2002). However, confetti-labelled CK14 ${ }^{+}$epithelial cells were shown to be the cells driven into the wound bed (Park et al., 2018), leading to the proposal of an alternative 'basal-cell theory' wherein basal cells are driven in this manner due to a population pressure gradient from the limbus toward the central cornea, due to enhanced proliferation in and around the LESC niche upon injury. Regarding the time scale, it is suggested from the results that such a proliferative response would begin by about 8 hours post-wounding. Data collected from mouse lineage tracing models would therefore indicate that following a brief latent period, the limbus is directly involved throughout the wound healing process, driving both the movement and repopulation of the basal epithelium.

Recent evidence would therefore support that not only is the limbus a key instrument of corneal epithelial wound healing through the generation of progenitors to replenish lost cells, but that more directly this process may drive a population pressure gradient forcing basal epithelial cells at the wound edge to extend into the wound bed. However, while this regenerative process would appear to be a key function of the limbus, the potential of basal corneal epithelial cells should perhaps not be discounted, and it would be particularly interesting to see clearer evidence of how factors like the size of an injury, or the age of tissue might influence the relative contributions of these cell types. 


\section{The LESC niche}

\subsection{The stem cell niche:}

The idea that stem cell preservation and function is dependent on their interaction with specific anatomical sites was first introduced in the study of haematopoietic stem cells and their relationship with the bone marrow (Schofield, 1978). The stem cell niche can be defined as specific microenvironment in which stem cells exist and can involve a range of factors including extracellular matrix, support cells, neurovascular inputs, and soluble signalling factors. The niche has been established as being essential to determining stem cell fate and directing the fate of their progeny as they leave the niche and differentiate into specific tissue cells. This is particularly well illustrated in the intestinal epithelium. Intravital imaging of Lrg5 confetti mice has revealed the short term dynamics of intestinal stem cells in the crypts (Ritsma et al., 2014). While many cells exhibit the potential to function as stem cells, only a small proportion fulfil this role, and this would appear to primarily be a function of the position of cells within the intestinal crypt niche (Walther and Graham, 2014).

\subsection{The limbal niche microstructure and components:}

A key objective has been to characterise the many components of the limbal epithelial stem cell niche and the degree to which they influence stem cell fate, and also link this fate to the changing needs of the corneal tissue.

When considering the features of the LESC niche, it is important to recognise the key differences in the limbal microenvironment, as compared the central cornea. One such difference is that unlike the uniform and flat corneal epithelial profile, the human limbus features palisades of Vogt, a series of fibrovascular ridges that form the limbal crypts that have been proposed to represent the primary LESC niche (Shortt et al., 2007). Other structures have also been identified in this region, including "limbal epithelial crypts" extending beyond the limbus (Dua et al., 2005), and focal stromal projections (Shortt et al., 2007). Reminiscent of the crypts housing intestinal stem cells, these structures facilitate the integration of signals from the various niche factors. It should however be noted that while these anatomical features are found in humans and also in pigs (Grieve et al., 2015; Notara et al., 2011), they do not appear to be present in other mammals, including mice (Li et al., 2017; Patruno et al., 2017). One possibility is that this species difference may relate to the amount of scleral show, and that these crypts offer greater protection for eyes in which the limbus more exposed, for example to UV light. Nevertheless, even in the absence it is likely that other fundamental niche factors are conserved and are integral to maintaining the LESC phenotype and function.

One such niche factor, and another key difference with the avascular cornea is the vascular supply to the limbus, arising from the episcleral arterial circle (Papas, 2003). Imaging studies by (Shortt et al., 2007) have revealed that not only do limbal arcades associate closely with the limbal crypts, but that that focal stromal projections that extend from the limbal stroma into the epithelium also contain a central vessel. This close association between these niche structures and the vascular supply therefore supports the view that this dedicated vascular supply is a key component on the limbal niche microenvironment, through the supply of factors that are required to support the metabolic needs of LESCs (Huang et al., 2015) Notably there are also number of cell types that are observed in the limbal stroma in close association with the limbal crypts, including immune cells (Vantrappen et al., 1985), that are believed to be important in LESC maintenance. Moreover, direct and indirect cell-cell 
interactions with stromal cells (Dziasko et al., 2014) such as melanocytes (Dziasko et al., 2015) and corneal stromal stem cells (CSSCs) (Kureshi et al., 2015), have been shown to play an important role in dictating the quiescence and activation of LESCs. The contribution of many of these has been previously reviewed elsewhere (Dziasko and Daniels, 2016). Further features that are thought to contribute to the LESC niche are the composition and mechanical properties of the extracellular matrix, as well as soluble factors, and recent developments in our understanding of these are discussed below.

\subsubsection{Extracellular matrix of the limbal niche:}

With an increasing range of extracellular matrix factors being identified, it has been clear that the composition of the limbus exhibits a number of features that distinguish it from the cornea. Notably, the basement membrane of the limbal epithelium has been shown lack the long form of Type XII collagen (Wessel et al., 1997), but to contain distinct Type IV collagen chains and additional laminins (Ljubimov et al., 1995), as well as tenascin C, which has shown to co-localise with $\mathrm{ABCG}^{+}$and $\mathrm{p} 3^{+}$basal epithelial cell clusters (SchlötzerSchrehardt et al., 2007; Yeung et al., 2008). Thus, a wide range of limbal-specific matrix components have been identified, and their interaction with cells within the niche have been explored (Polisetti et al., 2016). More recent studies have helped to identify further matrix components that may contributing to the limbal niche microenvironment, including hyaluronan.

Amniotic membrane is widely used clinically for ocular surface disease and has also been one of the substrates on which cultured limbal epithelial cells are transplanted. In the process of identifying the components of amniotic membrane that underpin it's therapeutic benefit, heavy chain hyaluronan/pentraxin 3 (HC-HA/PTX3) was identified the key matrix factor (Tseng, 2016). Using a reunion model of limbal niche cells and progenitor cells in Matrigel, it was shown that sphere generation was abrogated, and markers associated with a quiescent stem cell phenotye, CEBP $\delta$ (Barbaro et al., 2007) and Bmi-1 (Umemoto et al., 2006), were upregulated (Chen et al., 2015).

Hyaluronan (HA) is a high molecular weight glycosaminoglycan and a ubiquitous component of the extracellular matrix. It can be found in high and low molecular weight forms and is naturally synthesised in vertebrates by three different hyaluronan synthases (HAS), HAS1, HAS2, and HAS3 (Sun et al., 2019). Building on the work of Tseng and colleagues regarding HC-HA/PTX3, the role and distribution of endogenous HA expression has been explored in mouse models (Gesteira et al., 2017). The group report that in wild type unchallenged corneas HA is present solely in the limbal region, not present in the central cornea, and with weaker staining in the perilimbal conjunctiva. All three HAS enzymes are expressed in the limbus, and knockout or reduction in expression of each enzyme in turn, while not resulting in any macroscopic defects cause a reduction in the number of epithelial layers and altered basal cell morphology, and delayed wound healing was observed in each. In particular when HAS2 expression was significantly reduced in HAS2DcorEpi mice induced at $p 7$ there was a loss of $\mathrm{CK}_{15}{ }^{+}$cells in the limbus, and following alkali wounding, goblet cells were identified in the peripheral and limbal epithelium indicating conjunctivalisation. Therefore, it would appear that loss of HAS2 may manifest as a dysfunction of the LESC compartment, and that the HA plays a role in preserving the stem cell population. It is suggested that in normal tissue, as progenitors migrate from the HA-rich limbus, the loss of an HA environment might promote their differentiation. Additionally, following wounding, the expression of $H A$ extended into the cornea with a concomitant expression of CK15 in the corneal epithelium. 
While the results in this study focus on the period immediately after injury and it is not clear whether these changes revert following wound resolution, the group propose that in response to injury, a transient HA matrix is generated (through increased HAS expression), and this promotes a progenitor phenotype throughout the cornea.

Thus, although the role of the ECM may have once been deemed to be solely keeping cells together, its dynamic nature and active role in the control of cell behaviour is being increasingly recognised. Notably, molecules like HA are increasingly being looked to the development of improved scaffolds for the transplantation of tissue and cells (Yazdani et al., 2019).

\subsubsection{Mechanical properties of the niche:}

A relatively recently identified paradigm in the regulation of stem cells is the role of the compliance of the matrix microenvironment (inversely proportional to the elastic modulus, and therefore stiffness) in the control of stem cell differentiation (Engler et al., 2006). The stem cell niche may therefore exhibit a distinct biomechanical profile. The hypothesis that substrate compliance is relevance to LESC phenotype and function has since been explored, both in vitro and in vivo (Gouveia et al., 2019). Using Brillouin spectro-microscopy (BSM), the mechanical properties of live human corneas were analysed revealing a greater compliance in the limbus as compared with the central cornea, and also that the low shift softer limbal regions aligned well with the distribution of putative LESC markers.

In vitro and ex vivo studies were performed, in which the gel scaffolds or corneal tissue was treated with collagenase in a specified pattern. Cultured LESCs exhibited greater expression of CK15, and reduced CK3 in a pattern reflecting those of enzyme treatment, while the central regions of organ cultured corneas treated with collagenase also showed an increase expression of limbal markers in the basal epithelium - CK15 and ABCG2. In both instances, the collagen treated substrates showed a reduced expression and nuclear localisation of YAP, a mechanotransduction transcription factor which regulates the activity other transcriptional regulators including Wnt/ß-catenin and Sox9 which have been demonstrated to themselves be regulators of LESC markers (Menzel-Severing et al., 2018).

Collagenase treatment was also applied to a rabbit in vivo model, and in addition to showing that the changes in molecular marker expression and epithelial phenotype on treated corneal regions was consistent with observations ex vivo, it was observed that limbal regions subjected to alkali burns and subsequent collagenase treatment demonstrated a greater expression of limbal-specific markers than wounded tissue that had not been treated with collagenase.

This evidence would suggest that LESC phenotype can be modulated directly through manipulation of the mechanical properties of the surface on which they are growing. The group has therefore expressed interest in assessing whether this model of limbal niche reconstruction might be applied clinically.

\subsubsection{Soluble niche factors:}

A number of cell types have been shown to influence the growth and phenotype of LESCs in culture (Dziasko and Daniels, 2016). While some of these relationships may be driven by direct cell-cell communication, it is likely that there is paracrine interaction between the populations, and even autocrine influences within populations. Soluble factors and their receptors are therefore likely to exert an influence on the phenotype and activation state of LESCs within the niche. 
Of particular interest is the widely conserved Wnt signalling pathway. Wnt signalling is a complex system with three primary pathways, the best studied of which is the canonical Wnt signalling pathway, involving the engagement of Wnt with frizzled and Lrp5/6 receptors and resulting in the nuclear translocation of ß-catenin and activation of the Tcf/Lef transcription machinery. In particular, there is a well-documented role for Wnt-mediated control of stem cells that go through periods of quiescence, proliferation, and differentiation (Clevers et al., 2014).

A series of Wnt isoforms, receptors, and inhibitors have been shown to be upregulated in the limbal epithelial cells and subjacent stroma (Han et al., 2014; Kulkarni et al., 2010; Mei et al., 2014; Nakatsu et al., 2011). Previous studies in vitro have shown a functional role for canonical Wnt signalling in the promotion of LESC proliferation and the maintenance of a stem-like phenotype (Han et al., 2014; Lu et al., 2011; Mei et al., 2014; Nakatsu et al., 2011). More recently this work has been developed further with the use of targeted chemical modulators of canonical Wnt signalling to characterise the significance of this pathway on LESC maintenance (as determined by $\mathrm{p} 63 \alpha, \mathrm{CK} 12$, and CK14 expression) in vitro (González et al., 2019). Notably, unlike previous studies using molecules to interfere with intracellular steps of Wnt signalling, the use of IIIC3 and IC15 in this study specifically targets Wntreceptor interactions in the canonical pathway. The authors observed that at $5 \mu \mathrm{M}$ concentrations, activation of Wnt signalling with IIIC3 increased the number of cells in culture, and the proportion of which were $p 63 \alpha^{\text {bright }}$, while inhibition with IC15 resulted in a statistically significantly lower colony-forming efficiency (CFE). It will be important to identify which components of the Wnt signalling machinery are principally effecting these changes in LESC behaviour. Interestingly both IC15 and IIIC3 rely upon the mimicry or interference of Dickkopf (DKK) binding. Therefore, as the authors suggest, it is possible that the balance of Wnt signalling in the regulation of quiescence and activation states may be driven by the behaviour of the endogenous inhibitors like DKK.

In vitro studies present a picture in which activation of canonical Wnt signalling drives the maintenance of the stem/progenitor phenotype and increased proliferation in culture. However, the question of the functional significance of canonical Wnt signalling in the 3D limbal niche context remains. This is highlighted by the observation that the activation and inhibition of Wnt signalling has diametrically opposite effects on the proliferation and phenotype of limbal epithelial cells depending on whether they are cultured following isolation from tissue, or via outgrowth from limbal explants (Lee et al., 2017). In line with observations from previous studies, CHIR99021, a GSK-3 $\beta$ inhibitor and activation of $\beta$ catenin activity, supported the growth and stem/progenitor phenotype of isolated cells cultured in low-calcium medium, while the porcn inhibitor, IWP2, had a growth-inhibitory and differentiation-inducing effect on the same cells. Surprisingly however, increasing $\beta$ catenin activity with CHIR99021 reduced epithelial cell outgrowth and expression of p63 $\alpha$ and $A B C G 2$ under explant culture conditions, while IWP2 promoted outgrowth and expression of these markers. It is important to recognise that disruption of GSK-3 $\beta$ activity primarily targets the canonical Wnt signalling pathway, and the relative contributions of the non-canonical pathways should also be considered. A key difference between the two culture conditions is the presence of niche factors in the limbal explant that may play a role in determining how epithelial cells respond to these signals.

It will be important to further characterise the relationship between Wnt signalling and LESC fate, both within the context of other niche factors, and also using more targeted chemical modulators, to better delineate the relative contributions of different components of this 
very complex signalling network. One development that may help bridge this gap is the use of optimised and validated ex vivo organ culture systems in which the behaviour of limbal epithelial cells in relation to these niche factors can be studied in a context that better reflects the native microenvironment of these cells.

A further question regarding soluble factors like Wnt and their role in LESC fate regulation is the mechanism by which these signals are conveyed. In the first instance, whether the source of these factors is epithelial cells themselves acting in an autocrine manner, or whether signals are emanating from other cells within the niche. Furthermore, there is the question of the mechanism of communication between cells; extracellular vesicles and exosomes in particular are increasingly being implicated as key mediators of cell-cell communication (Jing et al., 2018). It has been demonstrated that active Wnt signalling can be induced through exosomal transport of Wnt proteins (Gross et al., 2012), and also that that exosome mediated Wnt signalling plays a role in wound healing in the skin (Zhang et al., 2015). Notably, as well as carrying protein, microRNAs represent an important cargo of exosomes, and recent study has identified a number of microRNAs that are enriched in the LESC population (Kalaimani et al., 2020). Moreover, the study reported that a number of these microRNAs are predicted to be associated with signalling pathways, including the Wnt signalling pathway.

\subsection{The significance of the stem cell niche:}

This relationship between stem cells and their niche is very important and where possible, each should always be considered in the context of the other. When it comes to think about how to wield LESCs therapeutically, it is becoming increasingly apparent that focusing on reconstituting the niche will be a critical factor in being able to restore a functional stem cell pool (Yazdanpanah et al., 2019). This approach holds within it the assumption that an intact niche is capable of restoring the stem cell phenotype.

This premise was explored in a mouse model in which the impact of an intact niche in the recovery from LESC depletion was tested (Nasser et al., 2018). Limbal cells expressing GFP under CK15-promoter control were shown to exist at a boundary between $\mathrm{CK}^{+}$conjunctival cells, and $\mathrm{CK}_{12} 2^{+}$central corneal cells. Using a CK14 ${ }^{+}$confetti lineage tracing system in the same model, the group observed that co-labelled cells were found at the margin of limbal stripes, suggesting that the GFP ${ }^{+}$cells represented LESCs actively involved in regenerating the cornea. Two conditions were studied; the first of which involved complete removal of the limbal epithelium, but the niche was left intact, while in the second the limbal epithelium was removed but the niche was also damaged. The authors observed that under the first set of conditions, the limbal epithelial could be restored, as demonstrated by the restoration of $\mathrm{CK}_{15}{ }^{+} \mathrm{GFP}^{+}$cells at the limbal margin. However, under the second set of conditions, the lack of an intact limbal niche meant that after roughly 30 days post-injury there was no evidence of $\mathrm{CK}_{15}{ }^{+} \mathrm{GFP}^{+}$recovery, and neovascularisation of the cornea had developed. The group concluded that rather than being due to conjunctival transdifferentiation, or regeneration by residual stem cells, this limbal epithelial restoration was a result of dedifferentiation of committed corneal epithelial cells. Furthermore, they propose that this process is dependent on an intact niche. The possibility that a small population of stem or progenitor cells existing within the central cornea may be responsible for this recovery is considered, but on the grounds that the required number of cell divisions would be unable to be achieved within the time frame of $\mathrm{CK} 15^{+} \mathrm{GFP}^{+}$recovery, transdifferentiation is proposed as the primary mechanism. 
While they consider that a small population of stem or progenitor cells may exist within the central cornea and driving this process, they deem it unlikely on the grounds that the required number of cell divisions would be unable to be achieved within the time frame of $\mathrm{CK}_{15}{ }^{+} \mathrm{GFP}^{+}$recovery, and that instead transdifferentiation is the primary mechanism.

This hypothesis has been challenged, primarily on the grounds that there is strong evidence supporting that the movement of epithelial cells is centripetal, and that repopulation of the limbus by corneal cells would require centrifugal motion (Park et al., 2019). However, there is also some evidence to suggest that the centrifugal movement of cells is a function of population pressure from the increasing limbal population due to proliferation (Park et al., 2018). Since this model assumes a population pressure gradient, and it should be considered that following limbal epithelial debridement the direction of this gradient is reversed, and division of peripheral basal corneal epithelial cells might account for the centrifugal movement of cells in this situation.

Importantly, the recovery of $\mathrm{CK}_{15} 5^{+}$population at the limbus provides evidence that the niche itself includes powerful signals that are capable of mobilising the inherent plasticity of epithelial cells in the immediate vicinity. Thus, if through the careful manipulation of niche factors, the LESC phenotype can be restored or recreated, this has important ramifications both for improving the culture and transplantation of LESCS, and the development of new therapeutic strategies to rescue this population in damaged eyes. 


\section{Stem cell heterogeneity - In pursuit of LESC markers}

\subsection{Targeting the right population:}

The Achilles heel of LESC study has long been the lack of a unified biomarker of bona fide stem cells. LESCs have been identified through characterisation of their nuclear-tocytoplasm ratio ( $\mathrm{N} / \mathrm{C} \geq 0.7$ ), label-retaining ability, clonal capacity, and side population (SP) phenotype. An ever-growing panel of putative biomarkers have been linked with LESCS through their influence on the preservation of these LESC features. This list includes regulators of the cell-cycle, cell adhesion molecules, cytoskeletal proteins, transporters, growth factors, and their receptors - these have been well described and summarised in a number of other reviews (Gonzalez et al., 2018; Guo et al., 2018).

One of the challenges is that while it is well accepted that the limbal epithelium contains LESCs, when cells are isolated for this region for transcriptomic analysis it is likely that the LESCS only represent a small proportion of this population. While more that $80 \%$ of cells in the limbal epithelium have been reported to stain positively for p63 (albeit at varying

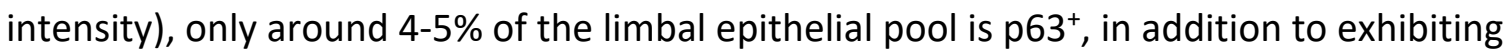
high N/C ratios, and retaining slow-cycling potential (Arpitha et al., 2008, 2005).

There have therefore been a number of recent marker-discovery models demonstrating ways in which a more representative LESC pool may be enriched and isolated for study. One such strategy is laser capture microdissection (LCM), in which cells can be selectively removed from tissue section based on features like size and position, providing an enriched pool which can be far more informative for downstream analysis (Bath et al., 2013; Kasinathan et al., 2016; Menzel-Severing et al., 2018; Polisetti et al., 2016). Another approach has been to purify a slow-cycling population of limbal epithelial cells using an inducible transgenic "pulse-chase" murine model, wherein the retention of a GFP label identifies quiescent cells within the tissue for co-expression studies and fluorescenceactivated cell sorting (FACS) for transcriptomic analysis (Sartaj et al., 2017).

\subsection{Revisiting putative markers:}

The $\mathrm{p} 63$ transcription factor, and the $\alpha$-isoform and $\Delta \mathrm{N}$-variant in particular, holds a prominent position among these markers having been shown not only to be a marker of holoclone cells (Pellegrini et al., 2001), but it has also been reported that the proportion of

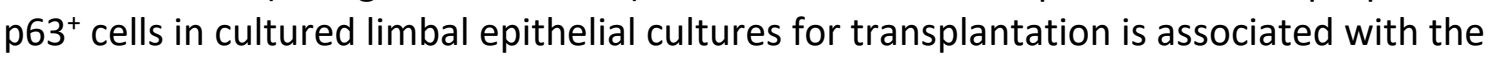
outcome of the procedure (Rama et al., 2010). Another often cited marker is the more recently characterised $A B C B 5$ transporter. Studies demonstrated that in addition to $A B C B 5$ being preferentially expressed on label-retaining LSC in mice and co-expressed with $\triangle \mathrm{Np63 \alpha}$-positive cells in humans, the expression of $A B C B 5$ was required for the restoration of an intact corneal epithelium in a mouse model of cultured limbal epithelial cell transplantation (Ksander et al., 2014). This novel marker was particularly attractive due to its membrane expression, meaning that it could offer a means by which limbal epithelial cell populations could be prospectively sorted to enrich the LESC population. The same group have also developed a mouse model in which the progeny of $\mathrm{ABCB} 5^{+}$mice can be traced, demonstrating that cells derived from this population can be found throughout the corneal epithelium (Gonzalez et al., 2018).

Despite the promise of $A B C B 5$, its expression in putative LESC populations has not always been reproduced, including in the $\mathrm{GFP}^{+}$slow-cycling pool of cells isolated from the inducible transgenic murine model (Sartaj et al., 2017). Furthermore, it has been raised that while 
ABCB5 knockout in mice manifested in abnormal corneal epithelial differentiation and wound healing, the presence of a corneal epithelium in these mice challenges the view of ABCB5 as a true marker of LESCs (Pellegrini et al., 2016).

As such the relative suitability of $A B C B 5$ and $p 63$ as surrogate markers of early progenitors was explored in human limbal epithelial cells (Liu et al., 2018). Cells were fluorescently labelled using antibodies against p63, ABCB5, and CK3, before being sorted into subpopulations using FACS. Of the sorted cells, four subpopulations were identified; $\mathrm{p} 63^{+} \mathrm{ABCB} 5^{+} \mathrm{CK}^{+}, \mathrm{ABCB}^{+}, \mathrm{p} 63^{+}$, and $\mathrm{p} 63^{+} \mathrm{ABCB} 5^{+}$. Next generation sequencing revealed that the greatest difference in transcriptomic profiles was between $\mathrm{p} 63^{+} \mathrm{ABCB} 5^{+} \mathrm{CK} 3^{+}$and $\mathrm{p} 63^{+}$ subpopulations, while the least difference was observed between the $p 63^{+}$and $p 63^{+} A B C B 5^{+}$ subpopulations. Furthermore, while the $\mathrm{p} 63^{+}$subpopulation uniquely had upregulation of a signature associated with pigmentation, the $A B C B 5^{+}$transcriptional signature more closely resembled the $\mathrm{p} 63^{+} \mathrm{ABCB} 5^{+} \mathrm{CK} 3^{+v e}$ subpopulation than the $\mathrm{p} 63^{+}$or $\mathrm{p} 63^{+} \mathrm{ABCB} 5^{+}$

subpopulations. The authors therefore conclude that this data supports a hierarchy in which ${\mathrm{p} 63^{+}}^{+}$represents a more immature progenitor, while ABCB5 marks progressively more committed precursors.

\subsection{Not all progenitors are equal:}

It is perhaps worth considering that one reason a unified marker has thus far eluded researchers is that LESCs are not a unified population. There is a growing body of evidence to indicate that cooperative stem cell subpopulations may exist in a wide range of adult tissues (Cheung and Rando, 2013; Li and Clevers, 2010). This is typified in the intestinal crypt epithelium wherein there is evidence to suggest that in anatomically distinct zones of the niche, active and quiescent stem cell pools exist and fulfil complementary roles in epithelial renewal and injury-induced regeneration (Bankaitis et al., 2018; Gehart and Clevers, 2019). An early indication of LESC subpopulations was the identification of C/EBP $\delta$ as regulator of mitotic activity, capable of inducing quiescence in $\triangle N p 63 \alpha^{+}$LESCs (Barbaro et al., 2007). One strategy that could be used to further interrogate these subpopulations is single-cell RNA sequencing, in that it enables the clustering and characterisation of relatively rare cell populations. Unbiased clustering of anterior murine ocular surface tissue has already been shown to be capable of detecting a range of distinct populations that are thought to include the stem cell pool, and separate early and late transient amplifying cell populations (Kaplan et al., 2019).

A recent screening analysis of 361 cell surface proteins via flow cytometry was performed on an expanded population of limbal epithelial cells proposing two novel markers, CD200 and CD109, as indicators of a quiescent and proliferative subpopulations (Bojic et al., 2018). Overlapping expression of both markers with $\Delta N p 63$ was observed, and expression of each decreased following differentiation. However, while the more abundant CD109+ population exhibited higher expression of Ki67, the much smaller CD200+ holoclone-generating population showed limited expression of $\mathrm{Ki} 67$ together with greater expression of the putative LESC markers $\triangle$ Np63, PAX6, WNT7A, CDH3, CK14, CK15, and ABCB5.

In contrast, in another study, CD200 expression was not detected in adult human or murine corneal sections and was only identified in developing murine corneas (Hayashi et al., 2018). Together with evidence that CD200 was widely expressed in non-epithelial colonies, and showed minimal expression in hPSC-derived corneal epithelial cells (notably only a small proportion, around $2 \%$, of primary limbal epithelial cells were found to be $C D 200^{+}$in the previous study), it was subsequently used a negative selective marker in the enrichment of 
hPSC-derived corneal epithelial cells from mixed populations. It should of course be kept in mind that while many features of corneal epithelial cells are present in hPSC-derived cultures, there may be significant differences with primary limbal epithelial cultures. In an attempt to probe the developmental hierarchy of LESCs, another study has made use of hPSC-derived cultures, tracking the in vitro differentiation of hPSC-derived corneal epithelial progenitors (Vattulainen et al., 2019). Interestingly, ABCG2, a putative LESC marker (de Paiva et al., 2005), was highlighted for its transient expression during the differentiation process. While there was apparent overlap between ABCG2 and p63 ( $\triangle \mathrm{Np63 \alpha}$ isoform) expression at different stages of the process, the authors observed that $A B C G 2^{+} p 63^{-}$cells were capable of generating a pure $A B C G 2^{-} \mathrm{p}^{-} 3^{+}$epithelial monolayer, placing $A B C G 2$ at an earlier position in the developmental hierarchy. Within the $A B C G 2^{+}$ populations, functionally distinct populations could be identified by expression of p63, namely a $\mathrm{p}^{-} 3^{-}$quiescent pool and a $\mathrm{p} 63^{+}$proliferative pool. Additionally, a cluster of p $63^{+} \mathrm{CK} 14^{+} \mathrm{CK} 15^{+}$cells emerged later in the proliferative process, proposed to represent an early transient amplifying progenitor pool. In addition to dissecting these populations, the group also demonstrated that the $\mathrm{ABCG}^{+}$subpopulations could be preserved in culture using an ENRC supplement - a collection of factors often used in the long term ex vivo organ culture of intestinal stem cells containing factors to promote Wnt signaling and dampen BMP activity (Leushacke and Barker, 2014). This observation might therefore reflect a mechanism through which niche signals in vivo are critical to the fate decisions of the LESC populations and exerting dynamic control of the developmental hierarchy. In the same study the expression of CD200 and CD109 was investigated in the ABCG2 ${ }^{+}$hPSCderived population. CD109 was expressed in a lower proportion of these cells as compared with primary LESCs, which the authors suggest may reflect the enhanced rate of differentiation in the hPSC-derived cultures. CD200, however, was expressed in a higher proportion of the hPSC-derived cells. Notably, the hPSC cultures in this study had undergone a much shorter period of differentiation than in the study performed by (Hayashi et al., 2018). As such it remains unclear whether CD200 represents a viable stem cell marker itself, and further investigation of this marker, particularly in primary epithelial cultures, is necessary.

Overall though, these results lend further support to the view indicate that LESC subpopulations may indeed be distinguishable from one another through differential expression of multiple markers, and that there is potential for a more functional hierarchy to be characterised.

\section{Conclusion}

The functional role of the limbus both in the maintenance and repair of the corneal epithelium is well accepted. However, a greater understanding of the limbal niche structure and the constituent factors is required to gain greater insight into how the cells of the limbus are able to respond appropriately to the dynamic needs of the tissue. Key to advancing this understanding is the delineation of the LESC developmental hierarchy, and the many putative LESC markers to identify functionally distinct subpopulations. A proposed outline of possible key limbal subpopulations is presented in (Figure 2), along with reference to how some of the key niche factors highlighted in this review may drive changes in cell fate. Overall, therefore, a key goal of this field should be to better establish the relationship between the niche factors and the behaviour of LESCs and their progeny. 


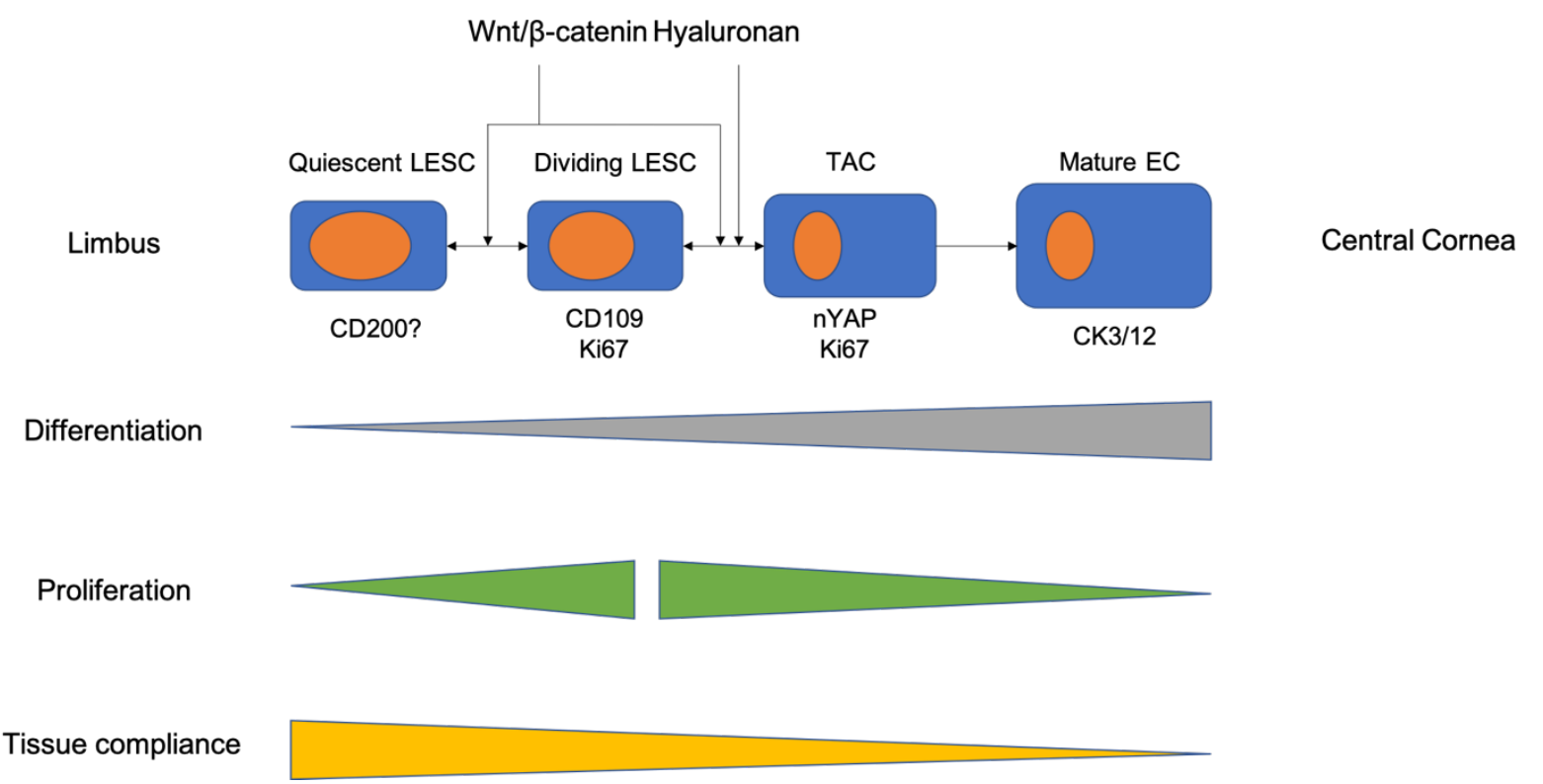

\section{Figure 2}

This diagram depicts a proposed scheme of the hierarchy of limbal progenitors, with quiescent LESCS representing the least differentiated form that may be labelled by CD200. These quiescent stem cells represent a small proportion of the limbal epithelial population that can shift between a slow-cycling state and an actively cycling state, labelled by markers or proliferation and CD109, in order to respond to the needs of the tissue. This interplay between the two subpopulations may be governed by the activity of niche signalling factors like Wnt/B-catenin. As cells divide and migrate centripetally out of the niche, signalling cues from soluble niche factors, niches cells and extracellular matrix components such as hyaluronan are lost. Together with altered mechanotransduction through the nuclear localisation of YAP, this transition may push cells toward a transient amplifying progenitor phenotype, which can repopulate the corneal surface with mature epithelial cells, as marked by CK3 and CK12. It is likely that further subdivision of each of these states that may be delineated through careful examination of previously identified and newly discovered putative markers of LESCS, in order to better characterise the fate of these cells. Notably, evidence from mouse models has also suggested that there is greater plasticity inherent in these cells, and that the right combination of niche factors is capable of dedifferentiating towards a developmentally earlier phenotype. $L E S C=$ limbal epithelial stem cell; TAC = transient amplifying progenitor; $E C=$ epithelial cell; $n Y A P=$ nuclear YAP transcription factor.

\section{Acknowledgements}

The authors would like to gratefully acknowledge the funding provided by the Rosetrees foundation that supported this work. 


\section{References}

Amitai-Lange, A., Altshuler, A., Bubley, J., Dbayat, N., Tiosano, B., Shalom-Feuerstein, R., 2015. Lineage tracing of stem and progenitor cells of the murine corneal epithelium. Stem Cells 33, 230-239. https://doi.org/10.1002/stem.1840

Arpitha, P., Prajna, N.V., Srinivasan, M., Muthukkaruppan, V., 2008. A subset of human limbal epithelial cells with greater nucleus-to-cytoplasm ratio expressing high levels of p63 possesses slow-cycling property. Cornea 27, 1164-1170. https://doi.org/10.1097/ICO.0b013e3181814ce6

Arpitha, P., Prajna, N.V., Srinivasan, M., Muthukkaruppan, V., 2005. High Expression of p63 Combined with a Large N/C Ratio Defines a Subset of Human Limbal Epithelial Cells: Implications on Epithelial Stem Cells. Invest. Ophthalmol. Vis. Sci. 46, 3631-3636. https://doi.org/10.1167/iovs.05-0343

Bankaitis, E.D., Ha, A., Kuo, C.J., Magness, S.T., 2018. Reserve Stem Cells in Intestinal Homeostasis and Injury. Gastroenterology 155, 1348-1361. https://doi.org/10.1053/j.gastro.2018.08.016

Barbaro, V., Testa, A., Di lorio, E., Mavilio, F., Pellegrini, G., De Luca, M., 2007. C/EBPdelta regulates cell cycle and self-renewal of human limbal stem cells. J. Cell Biol. 177, 1037-1049. https://doi.org/10.1083/jcb.200703003

Bath, C., Muttuvelu, D., Emmersen, J., Vorum, H., Hjortdal, J., Zachar, V., 2013. Transcriptional Dissection of Human Limbal Niche Compartments by Massive Parallel Sequencing. PLoS One 8. https://doi.org/10.1371/journal.pone.0064244

Bojic, S., Hallam, D., Alcada, N., Ghareeb, A., Queen, R., Pervinder, S., Buck, H., Lange, A.A., Figueiredo, G., Rooney, P., Stojkovic, M., Shortt, A., Figueiredo, F.C., Lako, M., 2018. CD200 Expression Marks a Population of Quiescent Limbal Epithelial Stem Cells with Holoclone Forming Ability. STEM CELLS 36, 1723-1735. https://doi.org/10.1002/stem.2903

Cenedella, R.J., Fleschner, C.R., 1990. Kinetics of corneal epithelium turnover in vivo. Studies of lovastatin. Invest. Ophthalmol. Vis. Sci. 31, 1957-1962.

Chen, S.-Y., Han, B., Zhu, Y.-T., Mahabole, M., Huang, J., Beebe, D.C., Tseng, S.C.G., 2015. HCHA/PTX3 Purified From Amniotic Membrane Promotes BMP Signaling in Limbal Niche Cells to Maintain Quiescence of Limbal Epithelial Progenitor/Stem Cells. Stem Cells 33, 3341-3355. https://doi.org/10.1002/stem.2091

Cheung, T.H., Rando, T.A., 2013. Molecular regulation of stem cell quiescence. Nature Reviews Molecular Cell Biology 14, 329. https://doi.org/10.1038/nrm3591

Clevers, H., Loh, K.M., Nusse, R., 2014. Stem cell signaling. An integral program for tissue renewal and regeneration: Wnt signaling and stem cell control. Science 346, 1248012. https://doi.org/10.1126/science.1248012

Cotsarelis, G., Cheng, S.Z., Dong, G., Sun, T.T., Lavker, R.M., 1989. Existence of slow-cycling limbal epithelial basal cells that can be preferentially stimulated to proliferate: implications on epithelial stem cells. Cell 57, 201-209.

Crosson, C.E., Klyce, S.D., Beuerman, R.W., 1986. Epithelial wound closure in the rabbit cornea. A biphasic process. Invest. Ophthalmol. Vis. Sci. 27, 464-473.

Danjo, Y., Gipson, I.K., 2002. Specific transduction of the leading edge cells of migrating epithelia demonstrates that they are replaced during healing. Exp. Eye Res. 74, 199204. https://doi.org/10.1006/exer.2001.1115 
de Paiva, C.S., Chen, Z., Corrales, R.M., Pflugfelder, S.C., Li, D.-Q., 2005. ABCG2 Transporter Identifies a Population of Clonogenic Human Limbal Epithelial Cells. Stem Cells 23, 63-73. https://doi.org/10.1634/stemcells.2004-0093

Di Girolamo, N., Bobba, S., Raviraj, V., Delic, N.C., Slapetova, I., Nicovich, P.R., Halliday, G.M., Wakefield, D., Whan, R., Lyons, J.G., 2015. Tracing the fate of limbal epithelial progenitor cells in the murine cornea. Stem Cells 33, 157-169. https://doi.org/10.1002/stem.1769

Dorà, N.J., Hill, R.E., Collinson, J.M., West, J.D., 2015. Lineage tracing in the adult mouse corneal epithelium supports the limbal epithelial stem cell hypothesis with intermittent periods of stem cell quiescence. Stem Cell Res 15, 665-677. https://doi.org/10.1016/j.scr.2015.10.016

Dua, H.S., Shanmuganathan, V.A., Powell-Richards, A.O., Tighe, P.J., Joseph, A., 2005. Limbal epithelial crypts: a novel anatomical structure and a putative limbal stem cell niche. Br J Ophthalmol 89, 529-532. https://doi.org/10.1136/bjo.2004.049742

Dziasko, M.A., Armer, H.E., Levis, H.J., Shortt, A.J., Tuft, S., Daniels, J.T., 2014. Localisation of Epithelial Cells Capable of Holoclone Formation In Vitro and Direct Interaction with Stromal Cells in the Native Human Limbal Crypt. PLOS ONE 9, e94283. https://doi.org/10.1371/journal.pone.0094283

Dziasko, M.A., Daniels, J.T., 2016. Anatomical Features and Cell-Cell Interactions in the Human Limbal Epithelial Stem Cell Niche. Ocul Surf 14, 322-330. https://doi.org/10.1016/j.jtos.2016.04.002

Dziasko, M.A., Tuft, S.J., Daniels, J.T., 2015. Limbal melanocytes support limbal epithelial stem cells in 2D and 3D microenvironments. Exp. Eye Res. 138, 70-79. https://doi.org/10.1016/j.exer.2015.06.026

Engler, A.J., Sen, S., Sweeney, H.L., Discher, D.E., 2006. Matrix elasticity directs stem cell lineage specification. Cell 126, 677-689. https://doi.org/10.1016/j.cell.2006.06.044

Gehart, H., Clevers, H., 2019. Tales from the crypt: new insights into intestinal stem cells. Nat Rev Gastroenterol Hepatol 16, 19-34. https://doi.org/10.1038/s41575-0180081-y

Gesteira, T.F., Sun, M., Coulson-Thomas, Y.M., Yamaguchi, Y., Yeh, L.-K., Hascall, V., CoulsonThomas, V.J., 2017. Hyaluronan Rich Microenvironment in the Limbal Stem Cell Niche Regulates Limbal Stem Cell Differentiation. Invest. Ophthalmol. Vis. Sci. 58, 4407-4421. https://doi.org/10.1167/iovs.17-22326

Gonzalez, G., Sasamoto, Y., Ksander, B.R., Frank, M.H., Frank, N.Y., 2018. Limbal Stem Cells: Identity, Developmental Origin and Therapeutic Potential. Wiley Interdiscip Rev Dev Biol 7. https://doi.org/10.1002/wdev.303

González, S., Oh, D., Baclagon, E.R., Zheng, J.J., Deng, S.X., 2019. Wnt Signaling Is Required for the Maintenance of Human Limbal Stem/Progenitor Cells In Vitro. Invest. Ophthalmol. Vis. Sci. 60, 107-112. https://doi.org/10.1167/iovs.18-25740

Gouveia, R.M., Lepert, G., Gupta, S., Mohan, R.R., Paterson, C., Connon, C.J., 2019. Assessment of corneal substrate biomechanics and its effect on epithelial stem cell maintenance and differentiation. Nature Communications 10, 1-17. https://doi.org/10.1038/s41467-019-09331-6

Grieve, K., Ghoubay, D., Georgeon, C., Thouvenin, O., Bouheraoua, N., Paques, M., Borderie, V.M., 2015. Three-dimensional structure of the mammalian limbal stem cell niche. Exp. Eye Res. 140, 75-84. https://doi.org/10.1016/j.exer.2015.08.003 
Gross, J.C., Chaudhary, V., Bartscherer, K., Boutros, M., 2012. Active Wnt proteins are secreted on exosomes. Nature Cell Biology 14, 1036-1045. https://doi.org/10.1038/ncb2574

Guo, Z.H., Zhang, W., Jia, Y.Y.S., Liu, Q.X., Li, Z.F., Lin, J.S., 2018. An Insight into the Difficulties in the Discovery of Specific Biomarkers of Limbal Stem Cells. Int J Mol Sci 19. https://doi.org/10.3390/ijms19071982

Haddad, A., 2000. Renewal of the rabbit corneal epithelium as investigated by autoradiography after intravitreal injection of 3H-thymidine. Cornea 19, 378-383. https://doi.org/10.1097/00003226-200005000-00024

Han, B., Chen, S.-Y., Zhu, Y.-T., Tseng, S.C.G., 2014. Integration of BMP/Wnt signaling to control clonal growth of limbal epithelial progenitor cells by niche cells. Stem Cell Research 12, 562-573. https://doi.org/10.1016/j.scr.2014.01.003

Hanna, C., O'brien, J.E., 1960. Cell Production and Migration in the Epithelial Layer of the Cornea. Arch Ophthalmol 64, 536-539. https://doi.org/10.1001/archopht.1960.01840010538009

Hayashi, R., Ishikawa, Y., Katayama, T., Quantock, A.J., Nishida, K., 2018. CD200 facilitates the isolation of corneal epithelial cells derived from human pluripotent stem cells. Scientific Reports 8, 1-11. https://doi.org/10.1038/s41598-018-34845-2

Huang, A.J., Tseng, S.C., 1991. Corneal epithelial wound healing in the absence of limbal epithelium. Invest. Ophthalmol. Vis. Sci. 32, 96-105.

Huang, M., Wang, B., Wan, P., Liang, X., Wang, X., Liu, Y., Zhou, Q., Wang, Z., 2015. Roles of limbal microvascular net and limbal stroma in regulating maintenance of limbal epithelial stem cells. Cell Tissue Res. 359, 547-563. https://doi.org/10.1007/s00441014-2032-4

Jing, H., He, X., Zheng, J., 2018. Exosomes and regenerative medicine: state of the art and perspectives. Translational Research 196, 1-16. https://doi.org/10.1016/j.trsl.2018.01.005

Kalaimani, L., Devarajan, B., Subramanian, U., Ayyasamy, V., Namperumalsamy, V.P., Veerappan, M., Chidambaranathan, G.P., 2020. MicroRNA Profiling of Highly Enriched Human Corneal Epithelial Stem Cells by Small RNA Sequencing. Scientific Reports 10, 1-10. https://doi.org/10.1038/s41598-020-64273-0

Kaplan, N., Wang, J., Wray, B., Patel, P., Yang, W., Peng, H., Lavker, R.M., 2019. Single-Cell RNA Transcriptome Helps Define the Limbal/Corneal Epithelial Stem/Early Transit Amplifying Cells and How Autophagy Affects This Population. Invest. Ophthalmol. Vis. Sci. 60, 3570-3583. https://doi.org/10.1167/iovs.19-27656

Kasinathan, J.R., Namperumalsamy, V.P., Veerappan, M., Chidambaranathan, G.P., 2016. A novel method for a high enrichment of human corneal epithelial stem cells for genomic analysis. Microsc. Res. Tech. 79, 1165-1172. https://doi.org/10.1002/jemt.22771

Ksander, B.R., Kolovou, P.E., Wilson, B.J., Saab, K.R., Guo, Q., Ma, J., McGuire, S.P., Gregory, M.S., Vincent, W.J.B., Perez, V.L., Cruz-Guilloty, F., Kao, W.W.Y., Call, M.K., Tucker, B.A., Zhan, Q., Murphy, G.F., Lathrop, K.L., Alt, C., Mortensen, L.J., Lin, C.P., Zieske, J.D., Frank, M.H., Frank, N.Y., 2014. ABCB5 is a limbal stem cell gene required for corneal development and repair. Nature 511, 353-357. https://doi.org/10.1038/nature13426

Kulkarni, B.B., Tighe, P.J., Mohammed, I., Yeung, A.M., Powe, D.G., Hopkinson, A., Shanmuganathan, V.A., Dua, H.S., 2010. Comparative transcriptional profiling of the 
limbal epithelial crypt demonstrates its putative stem cell niche characteristics. BMC Genomics 11, 526. https://doi.org/10.1186/1471-2164-11-526

Kureshi, A.K., Dziasko, M., Funderburgh, J.L., Daniels, J.T., 2015. Human corneal stromal stem cells support limbal epithelial cells cultured on RAFT tissue equivalents. Sci Rep 5, 16186. https://doi.org/10.1038/srep16186

Last, J.A., Thomasy, S.M., Croasdale, C.R., Russell, P., Murphy, C.J., 2012. Compliance profile of the human cornea as measured by atomic force microscopy. Micron 43, 12931298. https://doi.org/10.1016/j.micron.2012.02.014

Le, Q., Xu, J., Deng, S.X., 2018. Review. The diagnosis of limbal stem cell deficiency. Ocul Surf 16, 58-69. https://doi.org/10.1016/j.jtos.2017.11.002

Lee, H.J., Wolosin, J.M., Chung, S.-H., 2017. Divergent effects of Wnt/ $\beta$-catenin signaling modifiers on the preservation of human limbal epithelial progenitors according to culture condition. Scientific Reports 7, 1-11. https://doi.org/10.1038/s41598-01715454-x

Lehrer, M.S., Sun, T.T., Lavker, R.M., 1998. Strategies of epithelial repair: modulation of stem cell and transit amplifying cell proliferation. J. Cell. Sci. 111 ( Pt 19), 2867-2875.

Leushacke, M., Barker, N., 2014. Ex vivo culture of the intestinal epithelium: strategies and applications. Gut 63, 1345-1354. https://doi.org/10.1136/gutjnl-2014-307204

Li, J., Xiao, Y., Coursey, T.G., Chen, X., Deng, R., Lu, F., Pflugfelder, S.C., Li, D.-Q., 2017. Identification for Differential Localization of Putative Corneal Epithelial Stem Cells in Mouse and Human. Sci Rep 7. https://doi.org/10.1038/s41598-017-04569-w

Li, L., Clevers, H., 2010. Coexistence of Quiescent and Active Adult Stem Cells in Mammals. Science 327, 542-545. https://doi.org/10.1126/science.1180794

Liu, L., Nielsen, F.M., Emmersen, J., Bath, C., Østergaard Hjortdal, J., Riis, S., Fink, T., Pennisi, C.P., Zachar, V., 2018. Pigmentation Is Associated with Stemness Hierarchy of Progenitor Cells Within Cultured Limbal Epithelial Cells. Stem Cells 36, 1411-1420. https://doi.org/10.1002/stem.2857

Ljubimov, A.V., Burgeson, R.E., Butkowski, R.J., Michael, A.F., Sun, T.T., Kenney, M.C., 1995. Human corneal basement membrane heterogeneity: topographical differences in the expression of type IV collagen and laminin isoforms. Lab. Invest. 72, 461-473.

Lu, R., Bian, F., Zhang, X., Qi, H., Chuang, E.Y., Pflugfelder, S.C., Li, D.-Q., 2011. The $\beta$ catenin/Tcf4/survivin signaling maintains a less differentiated phenotype and high proliferative capacity of human corneal epithelial progenitor cells. The International Journal of Biochemistry \& Cell Biology 43, 751-759. https://doi.org/10.1016/j.biocel.2011.01.018

Majo, F., Rochat, A., Nicolas, M., Jaoudé, G.A., Barrandon, Y., 2008. Oligopotent stem cells are distributed throughout the mammalian ocular surface. Nature 456, 250-254. https://doi.org/10.1038/nature07406

Mei, H., Nakatsu, M.N., Baclagon, E.R., Deng, S.X., 2014. Frizzled 7 maintains the undifferentiated state of human limbal stem/progenitor cells. Stem Cells 32, 938945. https://doi.org/10.1002/stem.1582

Menzel-Severing, J., Zenkel, M., Polisetti, N., Sock, E., Wegner, M., Kruse, F.E., SchlötzerSchrehardt, U., 2018. Transcription factor profiling identifies Sox9 as regulator of proliferation and differentiation in corneal epithelial stem/progenitor cells. Scientific Reports 8, 1-18. https://doi.org/10.1038/s41598-018-28596-3 
Nakatsu, M.N., Ding, Z., Ng, M.Y., Truong, T.T., Yu, F., Deng, S.X., 2011. Wnt/ß-Catenin Signaling Regulates Proliferation of Human Cornea Epithelial Stem/Progenitor Cells. Invest Ophthalmol Vis Sci 52, 4734-4741. https://doi.org/10.1167/iovs.10-6486

Nasser, W., Amitai-Lange, A., Soteriou, D., Hanna, R., Tiosano, B., Fuchs, Y., ShalomFeuerstein, R., 2018. Corneal-Committed Cells Restore the Stem Cell Pool and Tissue Boundary following Injury. Cell Rep 22, 323-331. https://doi.org/10.1016/j.celrep.2017.12.040

Notara, M., Schrader, S., Daniels, J.T., 2011. The porcine limbal epithelial stem cell niche as a new model for the study of transplanted tissue-engineered human limbal epithelial cells. Tissue Eng Part A 17, 741-750. https://doi.org/10.1089/ten.TEA.2010.0343

Papas, E.B., 2003. The limbal vasculature. Cont Lens Anterior Eye 26, 71-76. https://doi.org/10.1016/S1367-0484(02)00054-1

Park, M., Richardson, A., Pandzic, E., Lobo, E.P., Lyons, J.G., Di Girolamo, N., 2019. Peripheral (not central) corneal epithelia contribute to the closure of an annular debridement injury. Proc Natl Acad Sci U S A 116, 26633-26643. https://doi.org/10.1073/pnas.1912260116

Park, M., Richardson, A., Pandzic, E., Lobo, E.P., Whan, R., Watson, S.L., Lyons, J.G., Wakefield, D., Di Girolamo, N., 2018. Visualizing the Contribution of Keratin-14+ Limbal Epithelial Precursors in Corneal Wound Healing. Stem Cell Reports 12, 14-28. https://doi.org/10.1016/j.stemcr.2018.11.014

Patruno, M., Perazzi, A., Martinello, T., Blaseotto, A., Di lorio, E., lacopetti, I., 2017. Morphological description of limbal epithelium: searching for stem cells crypts in the dog, cat, pig, cow, sheep and horse. Vet. Res. Commun. 41, 169-173. https://doi.org/10.1007/s11259-017-9676-y

Pellegrini, G., Dellambra, E., Golisano, O., Martinelli, E., Fantozzi, I., Bondanza, S., Ponzin, D., McKeon, F., De Luca, M., 2001. p63 identifies keratinocyte stem cells. Proc. Natl. Acad. Sci. U.S.A. 98, 3156-3161. https://doi.org/10.1073/pnas.061032098

Pellegrini, G., Lambiase, A., Macaluso, C., Pocobelli, A., Deng, S., Cavallini, G.M., Esteki, R., Rama, P., 2016. From discovery to approval of an advanced therapy medicinal product-containing stem cells, in the EU. Regen Med 11, 407-420. https://doi.org/10.2217/rme-2015-0051

Polisetti, N., Zenkel, M., Menzel-Severing, J., Kruse, F.E., Schlötzer-Schrehardt, U., 2016. Cell Adhesion Molecules and Stem Cell-Niche-Interactions in the Limbal Stem Cell Niche. Stem Cells 34, 203-219. https://doi.org/10.1002/stem.2191

Rama, P., Matuska, S., Paganoni, G., Spinelli, A., De Luca, M., Pellegrini, G., 2010. Limbal Stem-Cell Therapy and Long-Term Corneal Regeneration. New England Journal of Medicine 363, 147-155. https://doi.org/10.1056/NEJMoa0905955

Ritsma, L., Ellenbroek, S.I.J., Zomer, A., Snippert, H.J., de Sauvage, F.J., Simons, B.D., Clevers, $\mathrm{H}$., van Rheenen, J., 2014. Intestinal crypt homeostasis revealed at single-stem-cell level by in vivo live imaging. Nature 507, 362-365. https://doi.org/10.1038/nature12972

Sartaj, R., Zhang, C., Wan, P., Pasha, Z., Guaiquil, V., Liu, A., Liu, J., Luo, Y., Fuchs, E., Rosenblatt, M.I., 2017. Characterization of slow cycling corneal limbal epithelial cells identifies putative stem cell markers. Sci Rep 7, 3793. https://doi.org/10.1038/s41598-017-04006-y

Schlötzer-Schrehardt, U., Dietrich, T., Saito, K., Sorokin, L., Sasaki, T., Paulsson, M., Kruse, F.E., 2007. Characterization of extracellular matrix components in the limbal 
epithelial stem cell compartment. Experimental Eye Research 85, 845-860. https://doi.org/10.1016/j.exer.2007.08.020

Schofield, R., 1978. The relationship between the spleen colony-forming cell and the haemopoietic stem cell. Blood Cells 4, 7-25.

Seyed-Safi, A.G., Daniels, J.T., 2020. A validated porcine corneal organ culture model to study the limbal response to corneal epithelial injury. Experimental Eye Research 108063. https://doi.org/10.1016/j.exer.2020.108063

Shortt, A.J., Secker, G.A., Munro, P.M., Khaw, P.T., Tuft, S.J., Daniels, J.T., 2007. Characterization of the limbal epithelial stem cell niche: novel imaging techniques permit in vivo observation and targeted biopsy of limbal epithelial stem cells. Stem Cells 25, 1402-1409. https://doi.org/10.1634/stemcells.2006-0580

Sun, M., Puri, S., Mutoji, K.N., Coulson-Thomas, Y.M., Hascall, V.C., Jackson, D.G., Gesteira, T.F., Coulson-Thomas, V.J., 2019. Hyaluronan Derived From the Limbus is a Key Regulator of Corneal Lymphangiogenesis. Invest. Ophthalmol. Vis. Sci. 60, 10501062. https://doi.org/10.1167/iovs.18-25920

Sun, T.-T., Tseng, S.C., Lavker, R.M., 2010. Location of corneal epithelial stem cells. Nature 463, E10.

Thoft, R.A., Friend, J., 1983. The X, Y, Z hypothesis of corneal epithelial maintenance. Invest. Ophthalmol. Vis. Sci. 24, 1442-1443.

Tseng, S.C.G., 2016. HC-HA/PTX3 Purified From Amniotic Membrane as Novel Regenerative Matrix: Insight Into Relationship Between Inflammation and Regeneration. Invest Ophthalmol Vis Sci 57, ORSFh1-ORSFh8. https://doi.org/10.1167/iovs.15-17637

Umemoto, T., Yamato, M., Nishida, K., Yang, J., Tano, Y., Okano, T., 2006. Limbal epithelial side-population cells have stem cell-like properties, including quiescent state. Stem Cells 24, 86-94. https://doi.org/10.1634/stemcells.2005-0064

Van Buskirk, E.M., 1989. The anatomy of the limbus. Eye 3, 101-108. https://doi.org/10.1038/eye.1989.16

Vantrappen, L., Geboes, K., Missotten, L., Maudgal, P.C., Desmet, V., 1985. Lymphocytes and Langerhans cells in the normal human cornea. Invest. Ophthalmol. Vis. Sci. 26, 220225.

Vattulainen, M., Ilmarinen, T., Koivusalo, L., Viiri, K., Hongisto, H., Skottman, H., 2019. Modulation of Wnt/BMP pathways during corneal differentiation of hPSC maintains ABCG2-positive LSC population that demonstrates increased regenerative potential. Stem Cell Res Ther 10, 236. https://doi.org/10.1186/s13287-019-1354-2

Walther, V., Graham, T.A., 2014. Location, location, location! The reality of life for an intestinal stem cell in the crypt. The Journal of Pathology 234, 1-4. https://doi.org/10.1002/path.4370

Wessel, H., Anderson, S., Fite, D., Halvas, E., Hempel, J., SundarRaj, N., 1997. Type XII collagen contributes to diversities in human corneal and limbal extracellular matrices. Invest. Ophthalmol. Vis. Sci. 38, 2408-2422.

Yazdani, M., Shahdadfar, A., Jackson, C.J., Utheim, T.P., 2019. Hyaluronan-Based Hydrogel Scaffolds for Limbal Stem Cell Transplantation: A Review. Cells 8. https://doi.org/10.3390/cells8030245

Yazdanpanah, G., Haq, Z., Kang, K., Jabbehdari, S., Rosenblatt, M.L., Djalilian, A.R., 2019. Strategies for reconstructing the limbal stem cell niche. Ocul Surf 17, 230-240. https://doi.org/10.1016/j.jtos.2019.01.002 
Yeung, A.M.-H., Schlötzer-Schrehardt, U., Kulkarni, B., Tint, N.L., Hopkinson, A., Dua, H.S., 2008. Limbal Epithelial Crypt: A Model for Corneal Epithelial Maintenance and Novel Limbal Regional Variations. Arch Ophthalmol 126, 665-669. https://doi.org/10.1001/archopht.126.5.665

Zhang, B., Wang, M., Gong, A., Zhang, X., Wu, X., Zhu, Y., Shi, H., Wu, L., Zhu, W., Qian, H., $\mathrm{Xu}, \mathrm{W} ., 2$ 2015. HucMSC-Exosome Mediated-Wnt4 Signaling Is Required for Cutaneous Wound Healing. Stem Cells 33, 2158-2168. https://doi.org/10.1002/stem.1771 